\title{
Pre-Emptive Traffic Management for a Cluster-Based TDMA System in Vehicular Communications
}

\author{
Nyoman W. Prasetya ${ }^{1,2}$, Tsang-Ling Sheu ${ }^{1}$, Achmad Basuki², Muhammad Aziz Muslim² \\ ${ }^{1}$ Department of Electrical Engineering, National Sun Yat-sen University, Taiwan \\ ${ }^{2}$ Department of Electrical Engineering, Brawijaya University, Malang, Indonesia \\ Email:njom007@gmail.com, sheu@ee.nsysu.edu.tw, abazh@ub.ac.id,muh_aziz@ub.ac.id
}

How to cite this paper: Prasetya, N.W., Sheu, T.-L., Basuki, A. and Muslim, M.A. (2017) Pre-Emptive Traffic Management for a Cluster-Based TDMA System in Vehicular Communications. Journal of Computer and Communications, 5, 31-52. http://dx.doi.org/10.4236/jcc.2017.51004

Received: December 5, 2016

Accepted: January 3, 2017

Published: January 6, 2017

Copyright $\odot 2017$ by authors and Scientific Research Publishing Inc. This work is licensed under the Creative Commons Attribution International License (CC BY 4.0). http://creativecommons.org/licenses/by/4.0/

\section{Open Access}

\begin{abstract}
The IEEE $802.11 \mathrm{p}$ is a standard in a vehicular communication system, known as Wireless Access in Vehicular Environment (WAVE). An implementation of that standard as the MAC Protocol in a high-density of nodes in Vehicular Ad-Hoc Networks (VANETs) may create a performance drawback, in particular for packet loss and delay whenever collisions happen. Introducing Time Division Multiple Access (TDMA) schemes can improve the performance. However, TDMA scheduling is difficult to manage the case of high-density of traffic, the high mobility of vehicles, and dynamic network topology. This journal proposes a clustered-based TDMA by traffic priority in VANETs. The clustered traffic is defined as high and low traffic priority and embedded in TDMA MAC Header. The evaluation result obtained through NS3 Simulator shows that the proposed approach performed better in a high-density of nodes.
\end{abstract}

\section{Keywords \\ VANET, Cluster-Based TDMA, MAC, QoS, Priority}

\section{Introduction}

The current rapid development of wireless technology leads to higher wireless communications standards for Intelligent Transportation System (ITS). Those standards are mainly defined to be used in traffic safety and non-safety applications. Safety applications provide driver information about critical situations in advance and require strict reliability and delay. Non-safety applications improve the driving comfort and usually are more bandwidth sensitive. The communications in ITS are defined by the IEEE 1609 and IEEE 802.11p standards. Those 
standards establish an IEEE 802.11 Wireless Local Area Network (WLAN) communications system called Wireless Access in Vehicular Environments (WAVE).

WAVE uses a contention-based protocol which is not sensitive to mobility and changes of topology (VANET's characteristic). The disadvantage is the unbounded delay because of the random access to the medium like Carrier Sense Multiple Access with Collision Avoidance (CSMA/CA).

In another hand, TDMA is a scheduled-protocol which is highly sensitive to the network topology. Any change in network topology will require a reconfiguration of the TDMA frame. These changes are necessary to reduce the produced latency when the assignation of the time slots to the users is wrong in distributed systems. The advantage of scheduled based protocol is the reduced number of collisions.

To improve the transmission efficiency as the number of nodes is increased, recently cluster-based TDMA is proposed so that the disadvantages of TDMA will be decreased. Space for the development of the TDMA is still widely open such as improving the Quality of Service (QoS) by giving priorities to different types of traffics. At this time, Pre-Emptive Traffic Management for a ClusterBased TDMA System in Vehicular Communications is proposed.

This journal proposed a new scheme to increase the TDMA performance using scheduling that assigned priority to different types of traffic that separated it into two categories: high and low priority traffic. TDMA System will provide time slots based on traffic priority on a cluster-based communications. The priority information is embedded in the TDMA MAC header. The system will process high priority traffic until it reaches the threshold. After that the low priority traffic will be processed. A new scheme to perform inter-cluster communications also proposed with reserving some part of the TDMA time slots for doing the forwarding between vehicle coordinators (VCs). Inter-cluster communications will use intra-cluster communication for forwarding its packet to its coordinator before forwarded again to another cluster coordinator until the destination reached. Simulation has been performed to observe the impact of giving priority into the TDMA header of packet data comparing to WAVE. Table 1 shows the abbreviations list of the terminology to be used in this journal.

\section{Related Work}

\subsection{Vehicular Ad-Hoc Network (VANET)}

Vehicular Ad Hoc Networks (VANETs) are created by applying the principles of mobile ad hoc networks (MANETs) - the spontaneous creation of a wireless network for data exchange-to the domain of vehicles. They are a key component of intelligent transportations systems (ITS).

The types of applications are used in Car to Car (C2C) and Car to Infrastructure (C2I) communications which are in general received the name of $\mathrm{C} 2 \mathrm{X}$ communications. C2X communications are defined by the IEEE 1609 and IEEE 802.11p standards. Those standards establish a WLAN communications system 
Table 1. List of Abbreviations.

\begin{tabular}{cc}
\hline CSMA/CA & Carrier Sense Multiple Access with Collision Avoidance \\
DSRC & Dedicated Short Range Communications \\
ITS & Intelligent Transportations Systems \\
MANET & Mobile Ad-hoc Networks \\
OBU & On-Board Units \\
QoS & Quality of Service \\
RSU & Road Side Units \\
TDMA & Time Division Multiple Access \\
V2I & Vehicle to Infrastructure \\
V2V & Vehicle to Vehicle \\
VANET & Vehicular Ad-hoc Network \\
VC & Vehicle Coordinator \\
VN & Vehicular Node \\
WAVE & Wireless Access in Vehicular Environment \\
WLAN & Wireless Local Area Network \\
\hline
\end{tabular}

called WAVE. In a WAVE system, there are two types of devices: the Road Side Units (RSUs) and the On-Board Units (OBUs). An RSU is a WAVE device that operates at a fixed position (usually along the road transport network) that supports communication and data exchange with OBUs. An OBU is a mobile or portables WAVE device that supports information exchange with RSUs and other OBUs. Vehicular Ad-hoc Networks (VANETs) were seen as a mere oneto-one application of MANET principles, and they have since then developed into a field of research in their own right.

VANETs consist of moving vehicles that create very dynamic networks. VANET is one of the special types of Mobile Ad-hoc Networks (MANETs), but it does not have an existing infrastructure or even centralized administration. The VANET has two essential communications: Vehicle-to-Vehicle (V2V) and Vehicle-to-RSU (V2R). To support V2V and V2R communications, the United States Federal Communication Commission (FCC) dedicated $75 \mathrm{MHz}$ radio spectrums in the $5.9 \mathrm{GHz}$ band for Dedicated Short Range Communications (DSRC) spectrum [1]. In recent years, stakeholders started to investigate the usability of Long Term Evolution (LTE) as an alternative solution for vehicular networking applications [2] [3].

\subsection{IEEE 802.11p Wireless Access in Vehicular Environments (WAVE)}

IEEE 802.11p and IEEE 1609 is an approved amendment to the IEEE 802.11 standard to add WAVE, a vehicular communication system. It defines enhancements to 802.11 (the basis of products marketed as Wi-Fi) required supporting Intelligent Transportation Systems (ITS) applications. This includes data 
exchange between high-speed vehicles and between the vehicles and the roadside.

At some point, $802.11 \mathrm{p}$ was considered for dedicated short-range communications (DSRC), a U.S. Department of Transportation project based on the Communications, Air-interface, Long and Medium range (CALM) architecture of the International Organization for Standardization for vehicle-based communication networks, particularly for applications such as toll collection, vehicle safety services, and commerce transactions via cars. The ultimate vision was a nationwide network that enables communications between vehicles and roadside access points or other vehicles. This work built on its predecessor ASTM E2213-03 from ASTM International.

DSRC was developed as WLAN standard IEEE $802.11 \mathrm{p}$ for the wireless access in the vehicular environment (WAVE). Besides the IEEE 802.11p, there tends to be other IEEE working group 1609 [4] employed in vehicular communications with the extent of providing specifications for handling upper layers within the protocol suites such as application layer traffic flow optimization model for road operators [5]. Midya et al. [6] purposed a novel vertical handoff scheme by using RSU.

The fundamental channel access mechanism of WAVE using Enhanced Distributed Channel Access (EDCA) techniques can increase the successful probability of accessing channels. Chang et al. [7] propose a novel channel access scheme, called Earliest Deadline First based Carrier Sense Multiple Access (EDF-CSMA). Guo et al. [8] purposed a communication scheduling and control with platoon modeling and $\mathrm{Xu}$ et al. [9] proposed an FF-based performance model to study the time-varying behavior of vehicular networks by using numerical-method-based queuing analysis. However, an unpredictable delay could occur due to the characteristics of random access.

To support the high priority safety application in VANET, the Medium Access Control (MAC) protocol is designed to provide efficient broadcast services. For instance, HER-MAC [10] provided a reliable broadcast mechanism of safety message by utilizing service channel in a hybrid scheme using TDMA and CSMA. Other hybrid scheme improved time slots acquisition [11] and provided strict priority for QoS flows compared to best-effort flows [12]. However, unpredictable delay still could occur due to the characteristics of CSMA.

\subsection{Time Division Multiple Access (TDMA)}

Time division multiple access (TDMA) is a channel access method for shared medium networks. It allows several users to share the same frequency channel by dividing the signal into different time slots. The users transmit in rapid succession, one after the other, each using its own time slot. This allows multiple stations to share the same transmission medium (e.g. radio frequency channel) while using only a part of its channel capacity. Properties of VANET are variable network density, large-scale networks, a predictable mobility model and rapid topology changes.

Non cluster-based TDMA called Self-organized TDMA purposed by Rezazade et al. [13]. E-VeMAC [14] solved the parallel transmission problem in the VeMAC protocol [15] then modified by adding a collision flag [16]. Cooperative 
communications are one of many solutions to perform non cluster-based TDMA [17] [18]. Peng et al. [19] purposed a cooperative and opportunistic TDMA transmission. In two-ways traffic can be decreased with Prediction-based TDMA MAC protocol (PTMAC) [20]. However, each node manages its time slot by itself and if it takes very long time to schedule, it will not only introduce additional delay in the data transfer but also consume more energy.

Almalag et al. [21] proposed a TDMA Cluster-based MAC for VANETs (TC-MAC) that integrates the advantage of clustering and the TDMA slot reservation scheme thus efficiently avoid collisions and decrease the packet drop ratio. However, it only considered the one hop intra-cluster communication. The scheme proposed in [21] has extended by modifying the length of TDMA frames as presented in [22], where multi-hop intra-cluster communication is also discussed. For a multichannel network, Enhanced TDMA Cluster-based MAC (ETCM) [23] is purposed.

Since the role of Vehicle Coordinator (VC) is important in a cluster, many cluster algorithms for MANET and VANET are proposed [24]. The clustering algorithms need to be refined to take into account of nodes location, direction, and speed as well to enhance the stability.

\subsection{Cluster-Based Communications}

The cluster-based concept uses the location as a parameter to make a cluster. A cluster head needs to be selected to serve as the network coordinator in cluster-based TDMA. Wang et al. [25] purposed a VANET modeling and clustering design under practical traffic, channel and mobility conditions. Farooq et al. [26] purposed a cluster-based multicast routing protocol for military vehicle and the other hand a cluster can be formed by using RSU as a center [27] [28].

Employing clustering techniques in IEEE 802.11p collides when the number of nodes increases in a cluster. To improve the transmission efficiency as the number of nodes is increased, recently cluster-based TDMA (CBT) is proposed, such as in [29]. In intra-cluster communications, a VC (VANET Coordinator) is not pre-assigned, but it is elected through a simple transmit-and-listen scheme. The elected VC is responsible for allocating time slots for data exchange among its Vehicle Nodes (VNs). When two clusters are approaching, the CBT can quickly resolve the collisions by reassigning time slots in one of the clusters.

When the VN increases, the waiting time to elect VC is less than IEEE 802.11p. A TDMA time structure and MAC-frame format in slot 0 are depicted in Figure 1. Nguyen et al. [30] [31] proposed a new scheme to elect VC inherited the time division in TDMA frame and MAC-frame format [29] by modifying SAM using two time slots shown in Figure 2.

\section{Cluster Based TDMA System}

\subsection{Architecture}

Cluster-Based TDMA has two kinds of communication, intra-cluster and inter-cluster communications. Intra-Cluster is communications established be- 


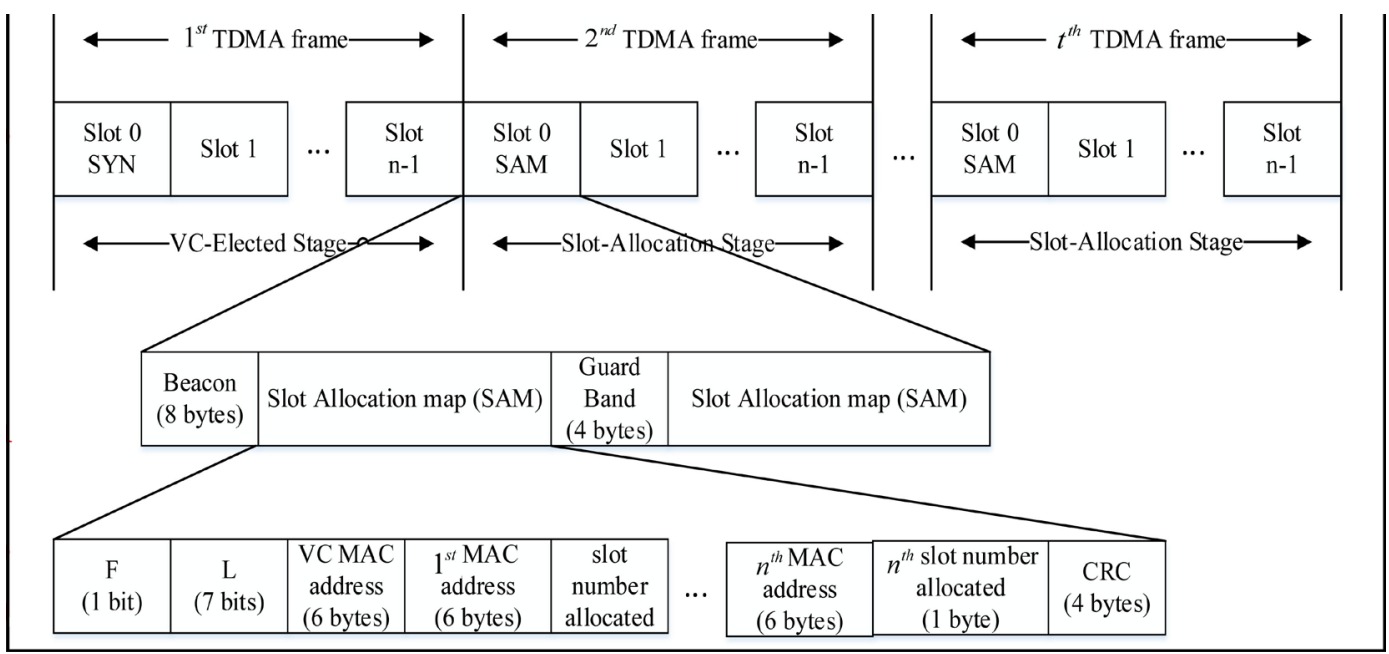

Figure 1. Time structure in [29].

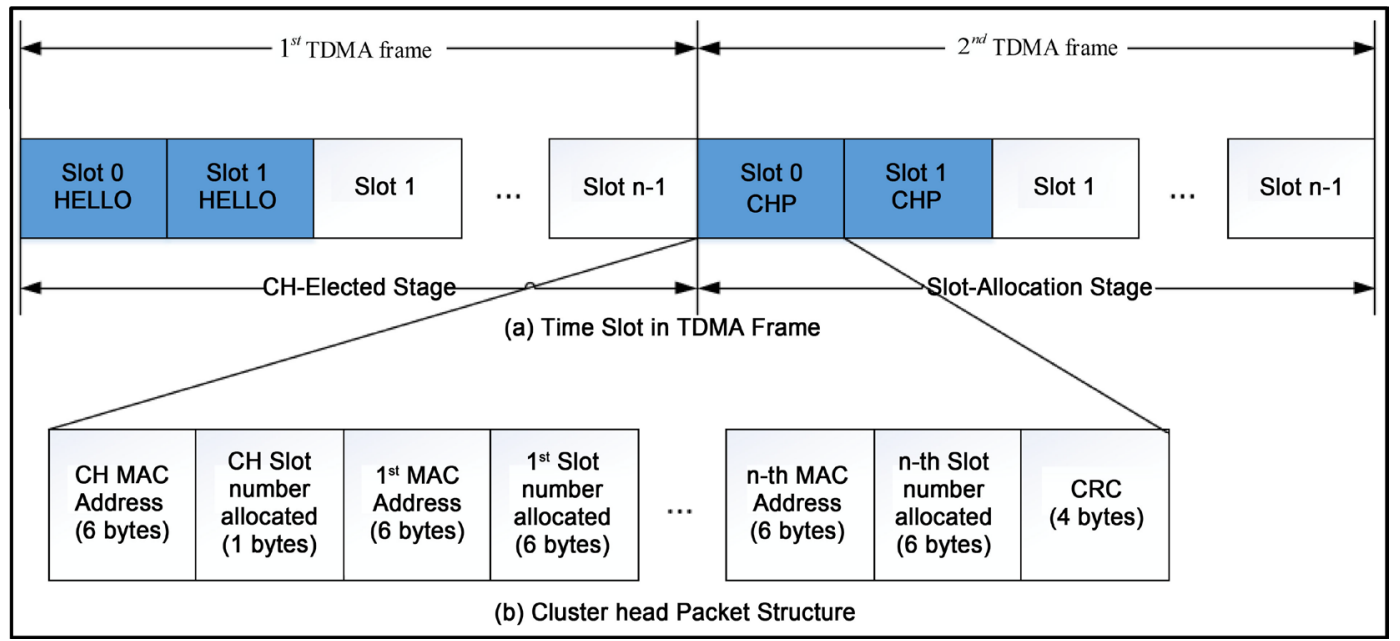

Figure 2. Time structure in [30].

ween members of a cluster. A cluster has a Vehicle Coordinator (VC) as a leader and has Vehicle Node (VN) as members. Coverage of a cluster is defined as the transmission range of $\mathrm{VC}$.

This cluster-based TDMA system uses the simple transmit-and-listen scheme to elect VC quickly and allow a VN to choose randomly a time slot for Bandwidth Request (BR) without limiting the number of VNs. In a cluster, VC announces its cluster information toward to VNs. Upon VNs receive VC's packet, VNs know about VC's MAC address and the other VNs in a cluster. VNs can transmit data together without collision by announcing a broadcast data packet on their assigned time slots. A cluster structure has one VC. In Figure 3, VC communicates with VNs within its transmission range.

Two VC of the two clusters possibly shares an overlapping area for certain time shown in Figure 4. In that case, two VC can coordinate about time slot assignment so members of a cluster can do exchanging information with members of the other cluster. The exchanging information with the other cluster makes an inter-cluster communications. 


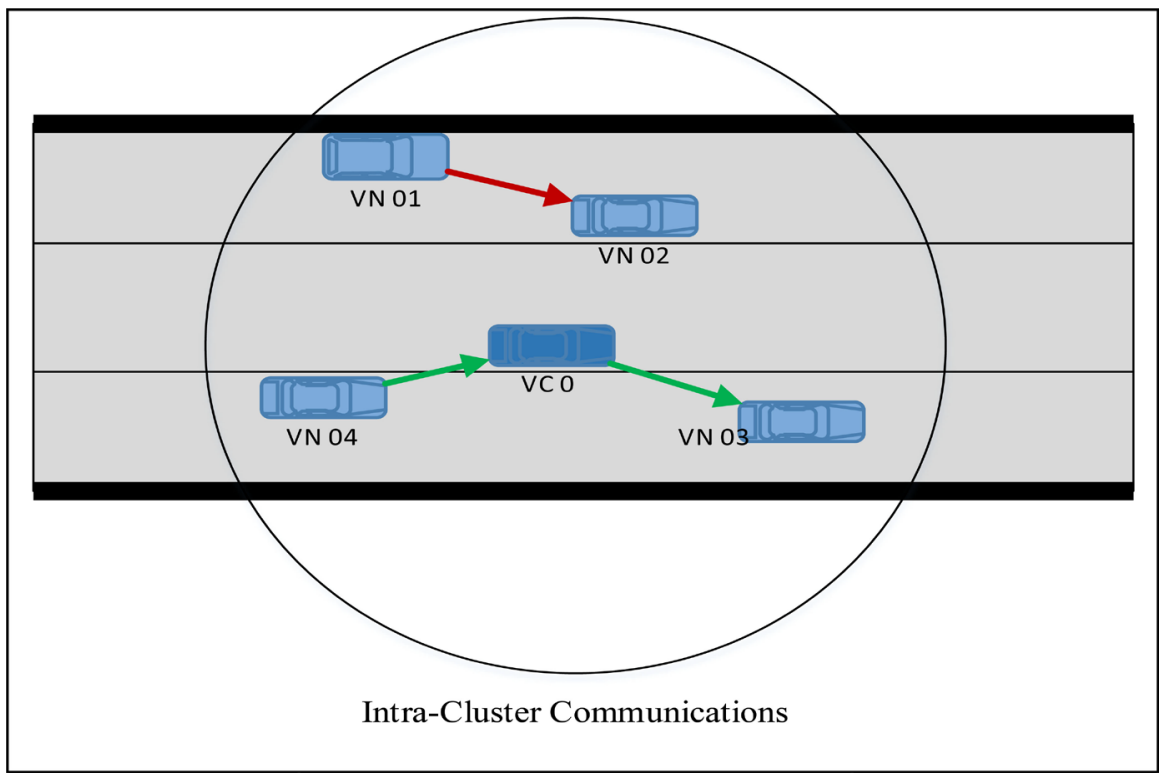

Figure 3. Intra-cluster communications.

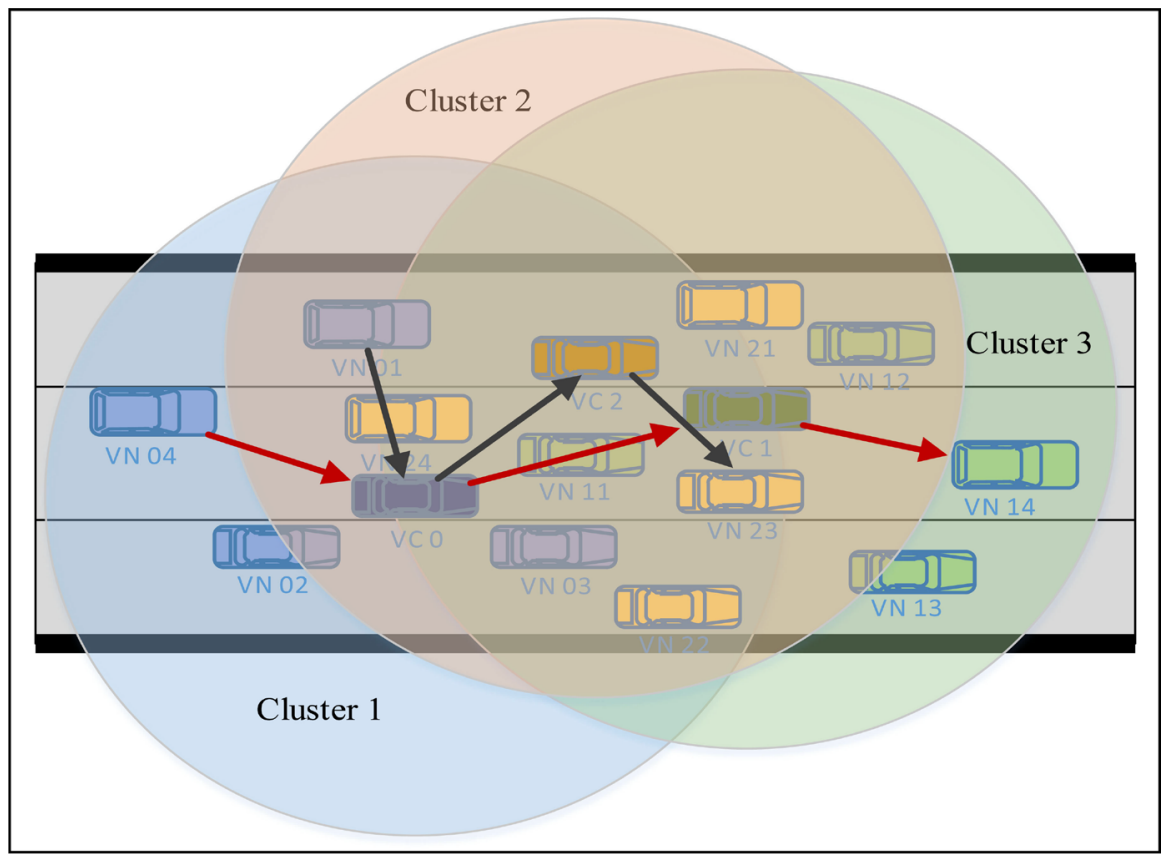

Figure 4. Inter-cluster communications.

\subsection{TDMA Header}

In this TDMA system, MAC frame format according to Sheu et al. [11] is used. The MAC frame format modified by adding 1 bit of priority field and 6 bits VC MAC address to the MAC header is shown in Figure 5. Priority field has two values, 0 indicate the high priority packet and 1 indicates low priority packet.

Six bits VC MAC address contains the coordinator's address to perform packet forwarding. In intra-cluster communication, packet forwarding occurs when the destination is out of range but still within the cluster, so the packet needs to be forwarded to the coordinator first before sent to the destination. In inter- 
cluster communication, packet forwarding occurs when clusters communicate. In this situation, 6 bits VC MAC address will contains next hop address.

\section{Pre-Emption in Cluster-Based TDMA}

Intra-cluster and inter-cluster communications have a different pre-emptive algorithm. Each communication process consists of three phases which are the initiation, pre-emption, and transmission.

\subsection{Priority}

To perform the simulation, we need to set the priority and the composition of the nodes that have high priority and low priority. In intra-cluster communications, half of the nodes will have the high priority (0) and the other half is low priority (1). In inter-cluster communications, the composition of the nodes is shown in Figure 6.

\subsection{Sort}

Intra-cluster and inter-cluster communications have a different pre-emptive algorithm. Each communication process consists of three phases which are the initiation, pre-emption, and transmission. In the pre-emptive algorithm, quicksort algorithm is used for sorting time slot request from VN according to its priority at request queue. Highest priority will be placed at the front of the queue and the

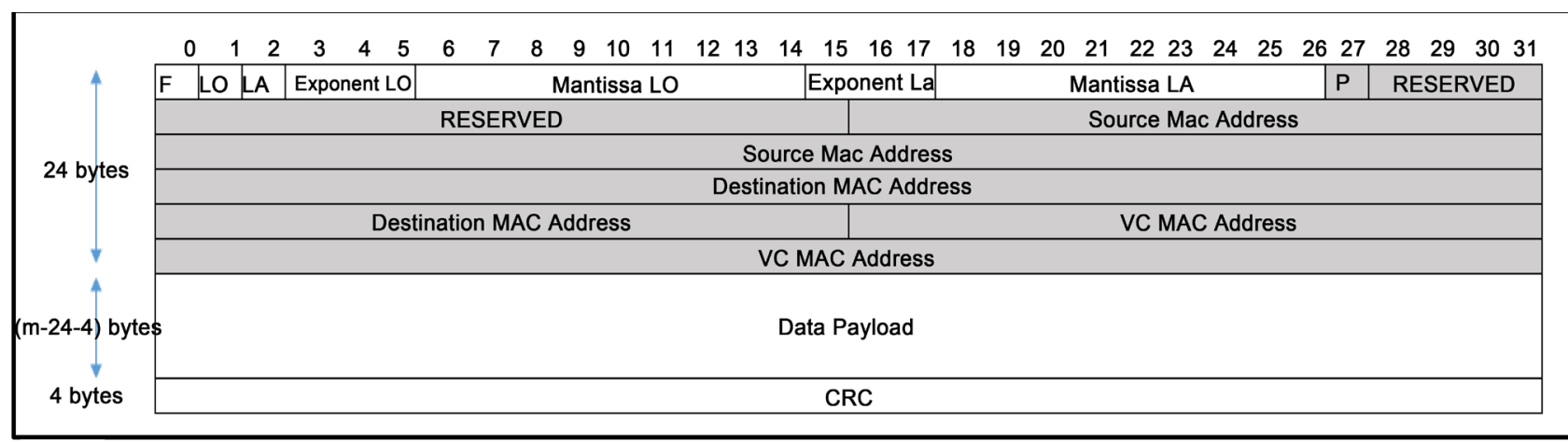

Figure 5. MAC header format.

\begin{tabular}{|c|c|c|c|c|}
\hline \multirow{3}{*}{ Inter-Cluster } & Cluster 1 & & Clust & \multirow{3}{*}{ Inter-Cluster } \\
\hline & $25 \%$ & High Priority & $25 \%$ & \\
\hline & $25 \%$ & Low Priority & $25 \%$ & \\
\hline \multirow{2}{*}{ Intra-Cluster } & $25 \%$ & High Priority & $25 \%$ & \multirow{2}{*}{ Intra-Cluster } \\
\hline & $25 \%$ & Low Priority & $25 \%$ & \\
\hline
\end{tabular}

Figure 6. Priority distribution. 
lower priority will be done sequentially.

Reverse Sort function for sorting the request of VN according to its priority in reverse at request queue uses quicksort algorithm. Lowest priority will be placed at the front of the queue, and the lower priority will be done sequentially.

\subsection{Accommodating}

After the request queue sorted by sort function, ten forefront request will get the time slots. This function is to flush the request queue to accommodate the new request later. This function used to remove the redundant request from the new request and unserved request from the previous frame. After the request queue accommodated by this function, it will be sorted by sort function.

\subsection{Intra-Cluster}

Initiation phase in intra-cluster begins by gathering information from every node (i.e. node id and its priorities). The information collected together and placed consecutively within a time slot array. In this phase, the counter will be given 0 as an initial value and pre-emption threshold is set to 2 and 5 .

On pre-emption phase, every VN sends a request for a time slot and all requests accommodated in the queue. At this time, the counter will be checked whether it is less than pre-emption threshold or not. If it so, sort function used to sort the nodes in request queue according to its priority. Sort function will put the higher priority on the front of the queue and then increment the counter. Otherwise, reverse sort function is used to sort the nodes in the request queue. Reverse sort function will put the lower priority on the front of the queue and then reset the counter to 0 . First 10 in the queue will be assigned to the intra-cluster time slots and make it into one TDMA frame.

On transmission phase, based on pre-emption threshold, the first TDMA frame transmitted is the highest priority frame and will be consecutively transmitted until the counter has the same value with pre-emption threshold. The lowest priority frame will be transmitted once before it starts to transmit the highest priority frame again shown in Figure 7.

\subsection{Inter-Cluster}

Initiation phase of inter-cluster begins with gathering information from every node such as node id and its priorities, and then putting that information together with time slot array of order. In this phase, the counter will be given 0 as

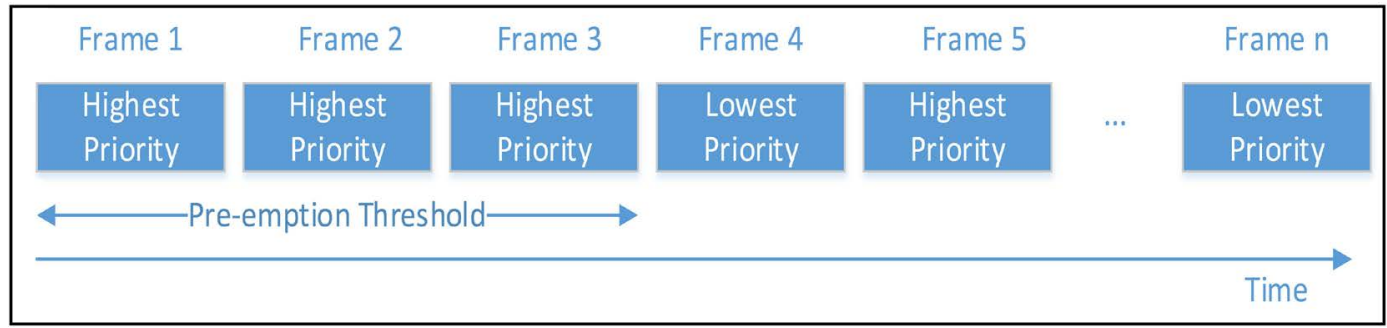

Figure 7. Intra-cluster frame scheduling. 
an initial value, and pre-emption threshold is set 2 and 5 .

In pre-emption phase, the nodes that do intra-cluster and inter-cluster communication will send a request for a time slot and accommodated in the request queue. Inter-cluster communications will use intra-cluster communication for forwarding its packet to its coordinator before forwarded again to another cluster coordinator to deliver it. At this time, counter value will be checked whether less than pre-emption threshold or not. If so, sort function is used to sort the nodes in request queue based on its priority and put the highest priority on the front of the queue and increment the counter. Otherwise, reverse sort function is used to sort the nodes in request queue based on its priority and put the lowest priority on the front side of the queue and then reset the counter to 0 . First 10 in the queue will be assigned to intra-cluster time slot to make one TDMA frame. Every cluster did this process before continuing to pre-emption phase for inter-cluster communications.

There are two steps in pre-emption phase for inter-cluster communication. The first step, VC buffer will be checked, whether there are packets to be sent to another cluster or not. At this time, the counter value will be checked again, whether less than pre-emption threshold or not. If so, sort function is used to sort the packets in VC's buffer according to its priority and put the highest priority on the front side of the buffer then increment the counter. If the counter is same or more than pre-emption threshold, reverse sort function used to sort the packets in VC's buffer according to its priority and put the lowest priority on the front of the buffer. First 6 of the VC's buffer will be recorded and request to Super VC will be sent. Super VC is a VC act as coordinator of VC. Super VC will check inter-cluster requests from all VCs.

The second step, Super VC will check the request queue for inter-cluster communications. Super VC will assign total 6 TDMA time slots for every VC to do inter-cluster communications. Super VC assigns the time slots for high priority request first. If the request has the same priority, time slots assignment will be in a round-robin manner.

After inter-cluster time slots formed, it needs to be combined with intracluster time slots to make TDMA frame. Total 16 TDMA time slots in one TDMA frame in the inter-cluster model consist of 10 intra-cluster slots at first and 6 inter-cluster time slots after that.

In transmitting phase, according to pre-emption threshold, the first TDMA frame transmitted will be the highest priority frame and consecutively transmit until the counter value is same or more than the pre-emption threshold. The lower priority frame will be transmitted once before it is starting to transmit the highest priority frame again shown in Figure 8.

\section{Simulation and Result}

\subsection{Simulation Program Architecture}

In intra-cluster communications, there are two kinds of communication will be performed. $\mathrm{VN}$ is able to communicate with another $\mathrm{VN}$ in the same cluster in 
direct communication or using VC to forward the data to another VN.

In inter-cluster communications, $\mathrm{VN}$ is able to communicate with another $\mathrm{VN}$ in another cluster. In this simulation, the packet from source VN will be forwarded to the VC of its cluster. VC will forward the packet to another VC of another cluster, then forward again to the destination VN.

\subsubsection{Intra-Cluster}

In intra-cluster communications, TdmaHelper function is an initiate function to send the first frame of TDMA. In this function IntraReq1 () function is called to check the communications request that sent by VN to the VC. Allocate2D () function is to allocate array and then put that request into an array and then sorted according to its priority by Sort () function and take the first ten on the list to send by calling SetDefaultSlots () function. Copy () function called to store the request list temporary, later this list will be compared to the new request list in the next TDMA frame to know whether there are a new VN or some VN left the cluster.

After the first TDMA frame sent, the module will call the Frame () function shown in Figure 9 to prepare and send the next TDMA frame. In Frame () func-

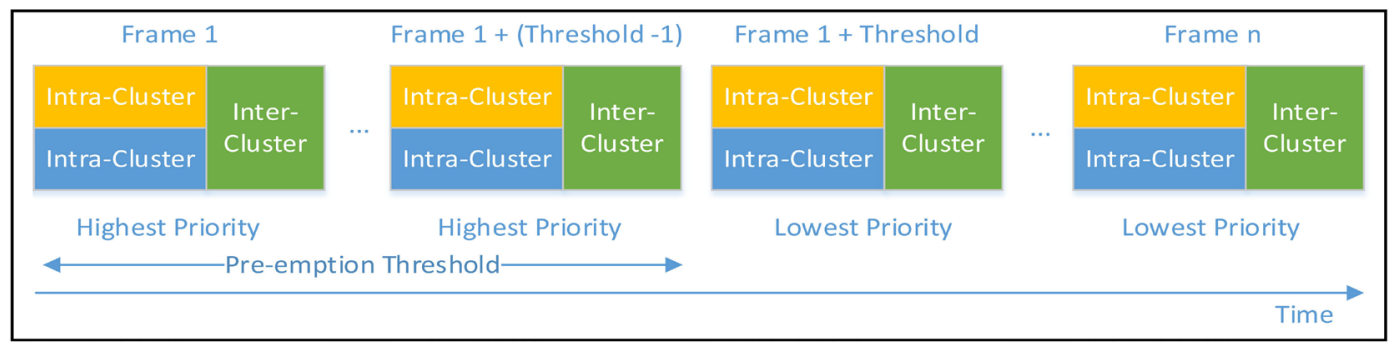

Figure 8. Inter-cluster traffic flow.

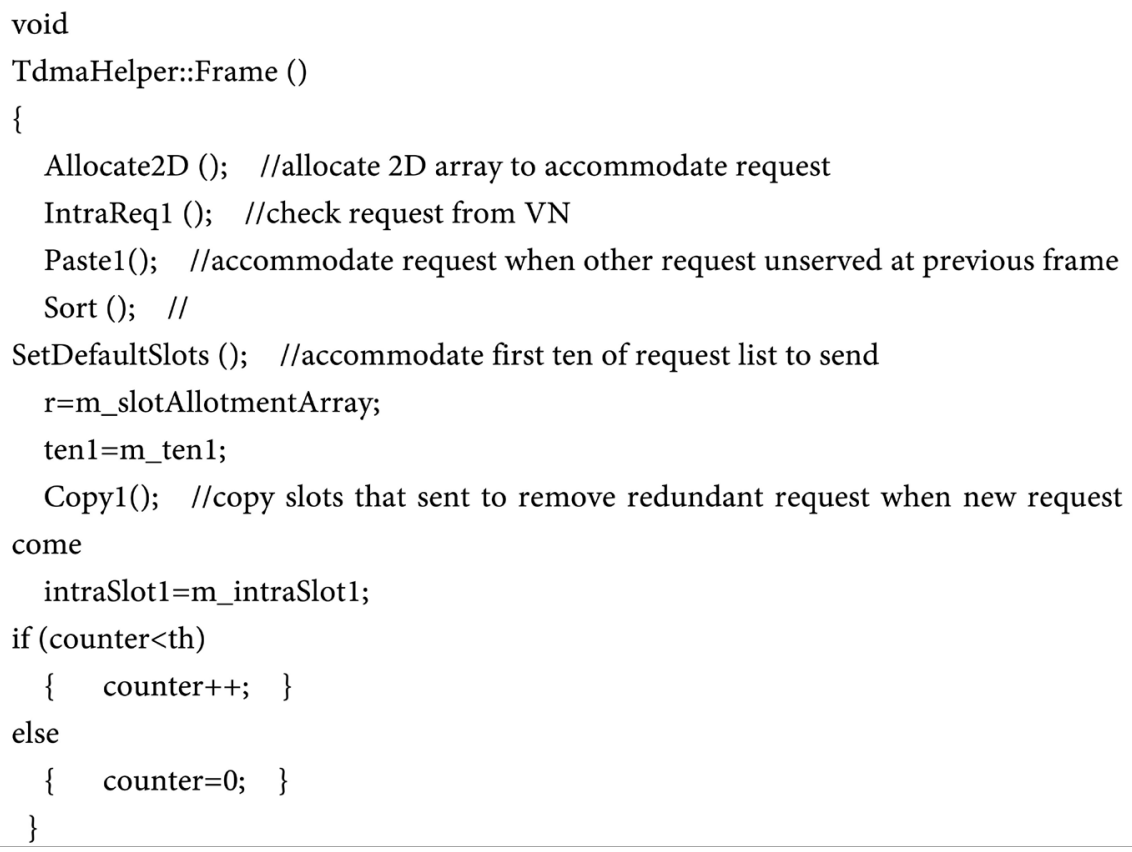

Figure 9. Intra-cluster frame function. 
tion, the new request in IntraReq1 () function will be compared with the old request in Copy () function, and then accommodate all the request by calling Paste1() function. After that, the request will be sorted again by Sort () function and take the first ten on the list to send. In Frame () function, the threshold will be checked. If the counter is less than the threshold, the counter will add the value by 1 or the counter value will be reset to 0 .

\subsubsection{Inter-Cluster}

In inter-cluster communications, TdmaHelper (), TdmaHelper2 () and TdmaHelper3 () function is an initiate function to send the first frame of TDMA for each cluster, just like intra-cluster communications. In this function IntraReq1 () (cluster 1) and IntraReq2 () (cluster 2) function is called to check the communications request that sent by VN to the VC. Allocate2D () (cluster 1) and AllocateArray () (cluster 2) function is to put that request into an array and then sorted according to its priority by Sort () (cluster 1) and Sort2 () (cluster 2) function and take the first ten on the list to send. Copy () (cluster 1) and Copy2 () (cluster; 2) function called to store the request list temporary, later this list will be compared with the new request list in the next TDMA frame to know whether there are a new VN or some VN left the cluster.

When TdmaHelper () function in process, it will call TdmaHelper2 () function to process the other cluster. AllocateCombine () function is to allocate an array to put the time slots order from all the cluster together. SetCombine () function will combine the time slots order.

After the first TDMA frame sent, the module will call the Frame () function shown in Figure 10 to prepare and send the next TDMA frame. At this stage, the process is similar to intra-cluster communication. The difference is in inter-cluster communication it will process two different clusters and in the end, it will combine together. In Frame () function, the threshold will be checked. If the counter is less than the threshold, the counter will add the value by 1 or the counter value will be reset to 0 .

\subsection{Simulation Parameter and Result}

This section explains about two simulations used by using Network Simulation 3 (NS3). The inter-cluster simulation will perform both intra-cluster and intercluster communications. This simulation used to evaluate intra-cluster and inter-cluster performance using parameters as shown in Table 2.

To perform intra-cluster and inter-cluster communications, we consider the scenario as a segment of one-way highway traffic. Each vehicle communicates within its communication range by sending UDP packets. The number of vehicles on the highway remains constant during the simulation time. In this section, we set all VN inside VC's transmission range.

\subsubsection{Intra-Cluster Simulation}

In this intra-cluster model, half of the nodes sent high priority packets and the other half sent low priority packets. In this simulation also perform simulation 


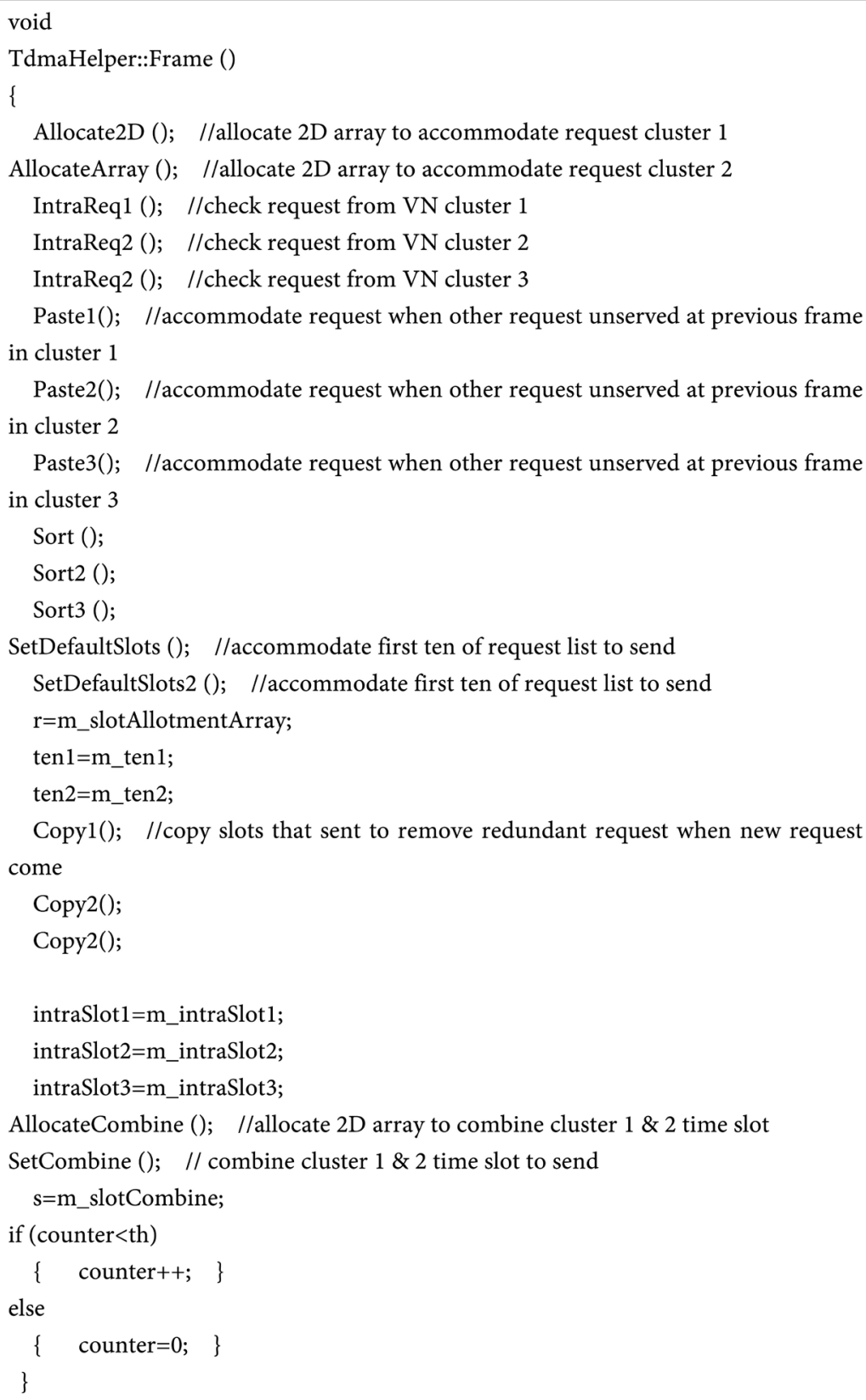

Figure 10. Intra-cluster frame function.

Table 2. Simulation parameter.

\begin{tabular}{ccc}
\hline Parameter & Intra-Cluster & Inter-Cluster \\
\hline Number of nodes & $10,20,30,40,50,60$ & $30,45,60,75$ \\
Number of time slots & 10 & 16 \\
Speed & $10 \mathrm{~km} / \mathrm{h}(\approx 3 \mathrm{~m} / \mathrm{s})$ to & $10 \mathrm{~km} / \mathrm{h}(\approx 3 \mathrm{~m} / \mathrm{s})$ to \\
& $20 \mathrm{~km} / \mathrm{h}(\approx 6 \mathrm{~m} / \mathrm{s})$ & $20 \mathrm{~km} / \mathrm{h}(\approx 6 \mathrm{~m} / \mathrm{s})$ \\
Packet size & $1000 \mathrm{bytes}$ & $1000 \mathrm{bytes}$ \\
Slot duration & $770 \mu \mathrm{sec}$ & $770 \mu \mathrm{sec}$ \\
Data rate & $1 \mathrm{Mbps}$ & $1 \mathrm{Mbps}$ \\
Threshold & $1,3,5$ & $1,3,5$ \\
Transmission range & $150 \mathrm{~m}$ & $150 \mathrm{~m}$ \\
\hline
\end{tabular}


using $802.11 \mathrm{p}$ (WAVE) as a comparison for TDMA by using the same parameters.

Figure 11 illustrates the average queue length of the simulation result. The length of the queue increases as the number of nodes increases, especially in WAVE. In WAVE the rapidly increasing of the queue length caused by random back off time of CSMA/CA mechanism. In TDMA, the average queue length of low priority nodes increased when the threshold is increased (high threshold).

In another hand the average queue length of the high priority nodes is decreased. The increasing of the threshold will raise the number of high priority time slots to be processed. This will make the queue of high priority nodes emptied faster but will make the queue of low priority nodes slower.

The result in Figure 12 shows that the average queuing delay increases along with increasing the number of nodes. According to the result, the delay of the high priority communications that use high threshold is lower than high priority communications that use low threshold. And the other hand, the delay of the low priority communications that using high threshold is higher than low priority communications that using low threshold. This is happened because of the increasing of the threshold will raise the number of high priority time slots will be processed by sacrificing the low priority time slots.

As shown in Figure 13, the packet loss is increased because of congestion as the number of nodes increased. WAVE performance falls on highest packet loss in a higher number of nodes. High priority TDMA showed the best performance by lowest packet loss, but the low priority TDMA experienced higher packet loss. This is happened because the low priority TDMA been sacrificed for better performance of the high priority TDMA.

The result in Figure 14 illustrates that the throughput decreases as the number of nodes increase. The performance of TDMA surpasses the WAVE starts

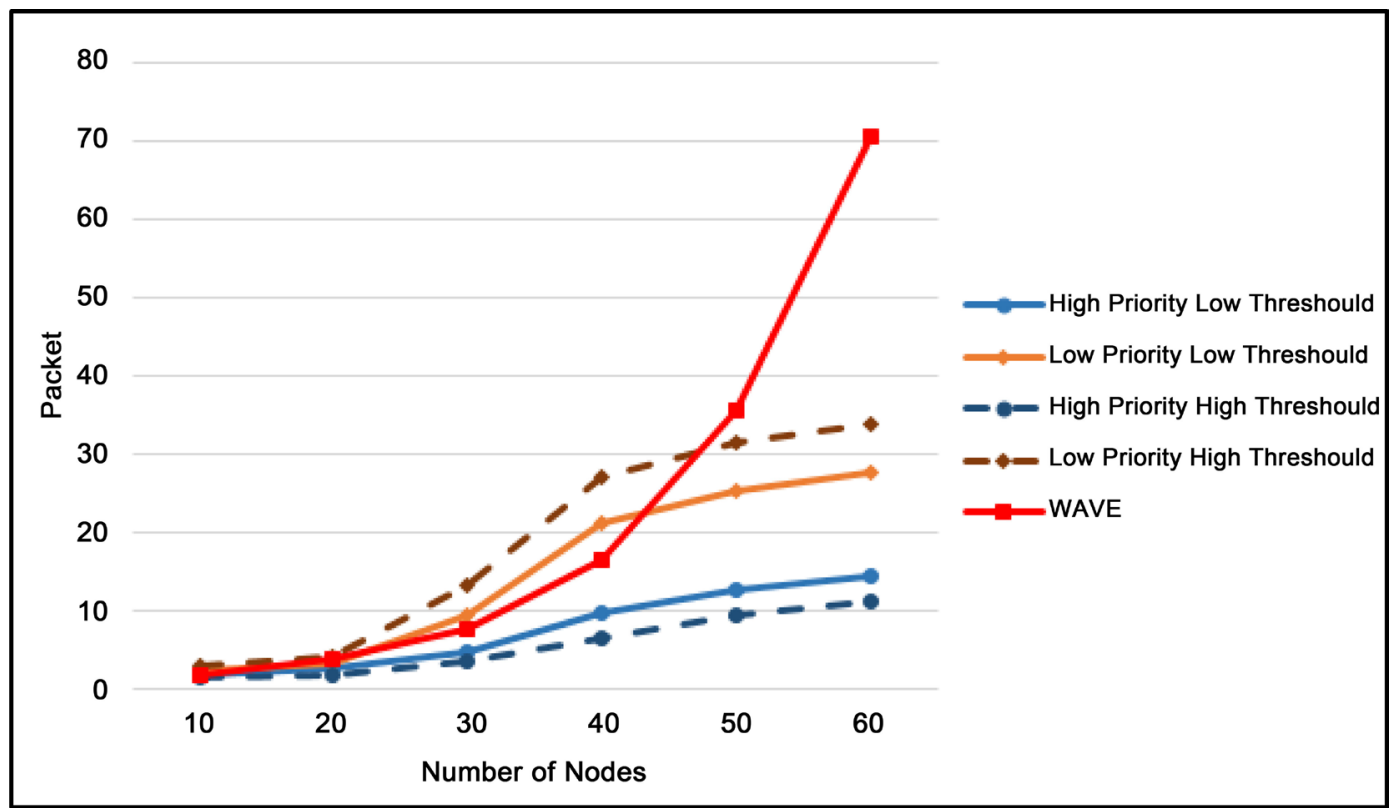

Figure 11. Intra-cluster average queue length. 


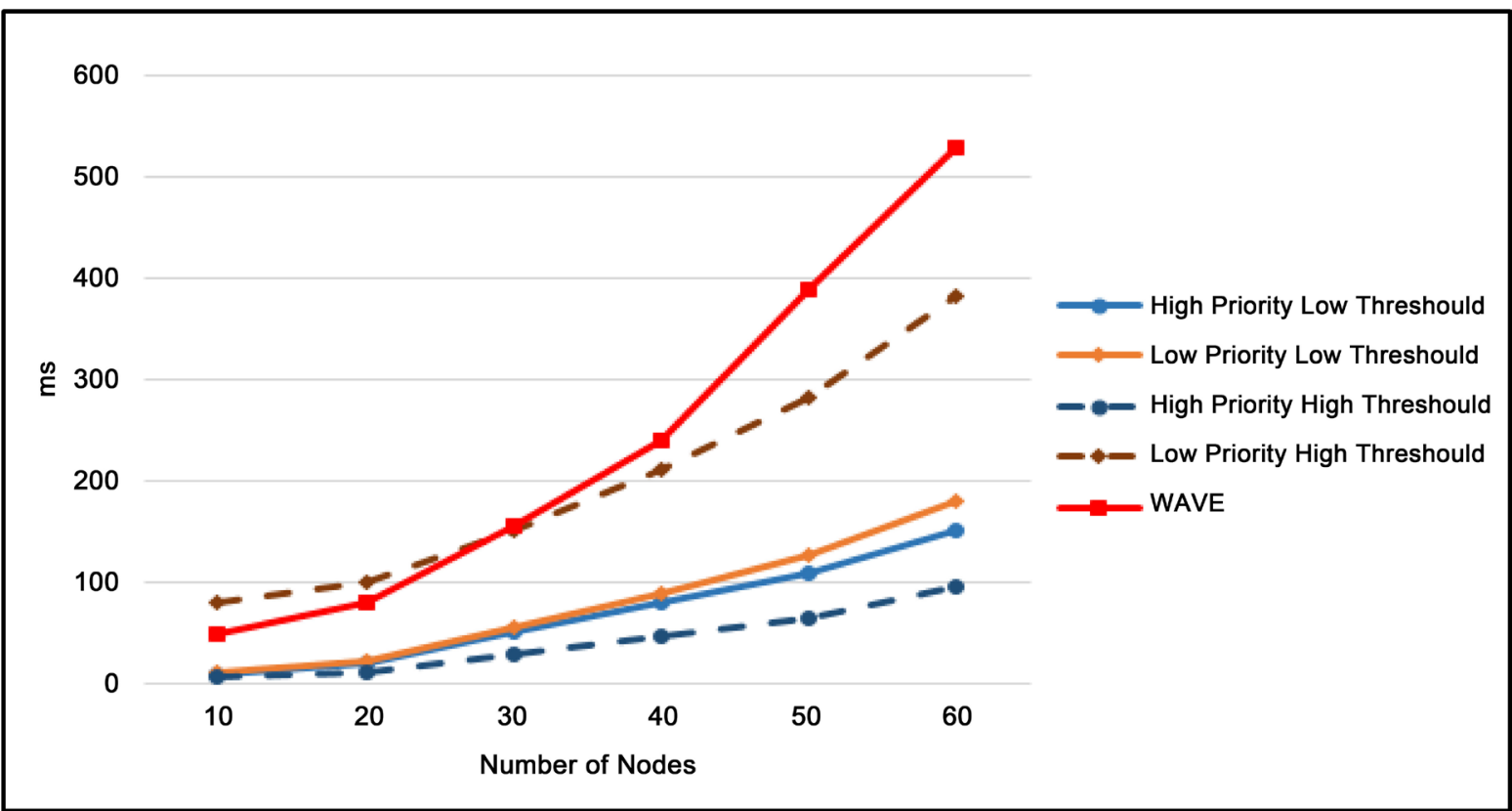

Figure 12. Average queuing delay in intra-cluster communications.

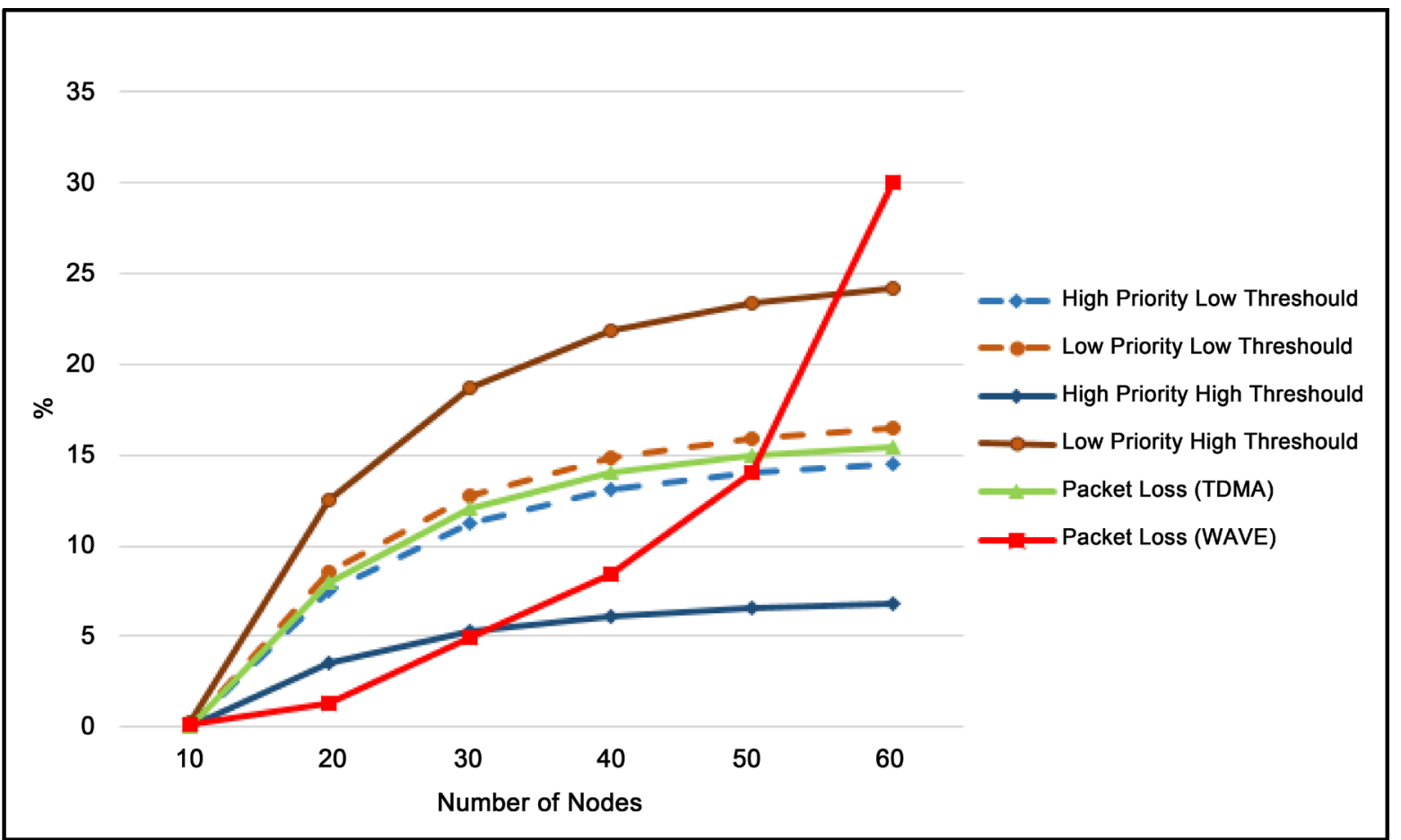

Figure 13. Packet loss ratio in intra-cluster communications.

from 40 nodes, but the performance of high priority TDMA surpasses all of the access methods start from 30 nodes. On the other side, the low priority TDMA surpasses the WAVE performance start from 50 nodes.

\subsubsection{Inter-Cluster Simulation}

In inter-cluster communication simulation, the number of nodes is divided into three clusters. $50 \%$ nodes in each cluster will do the intra-cluster communica- 
tion. Half of it sends high priority packets while the others send low priority packets. Another 50\% nodes in each cluster will do inter-cluster communication. Half of it sends high priority packets while others send low priority packets.

Average queue length of the simulation results shown in Figure 15. The length of the queue increases as the number of nodes increases. Even the low priority that been sacrificed for better performance of high priority is still has a

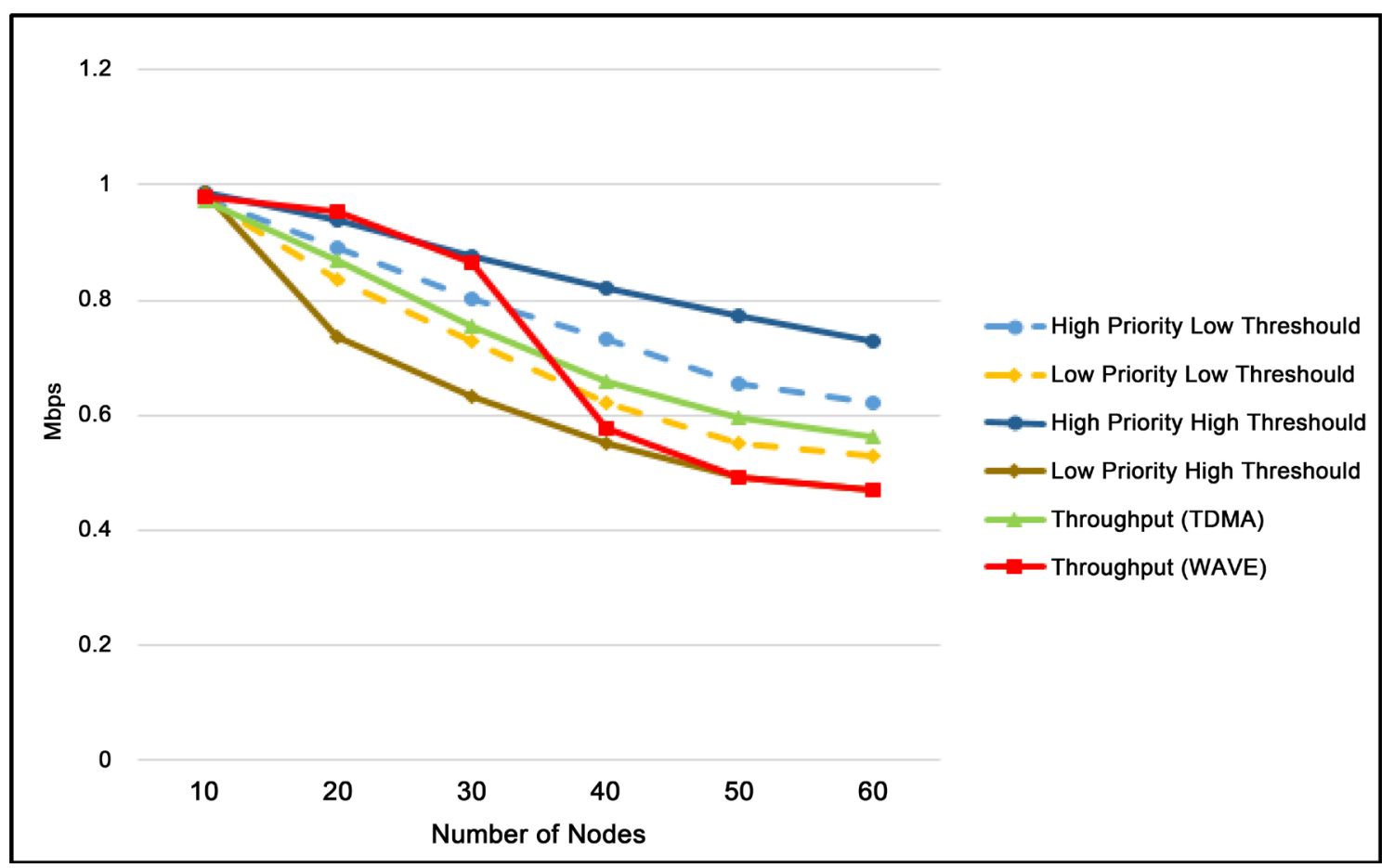

Figure 14. Throughput of intra-cluster communications.

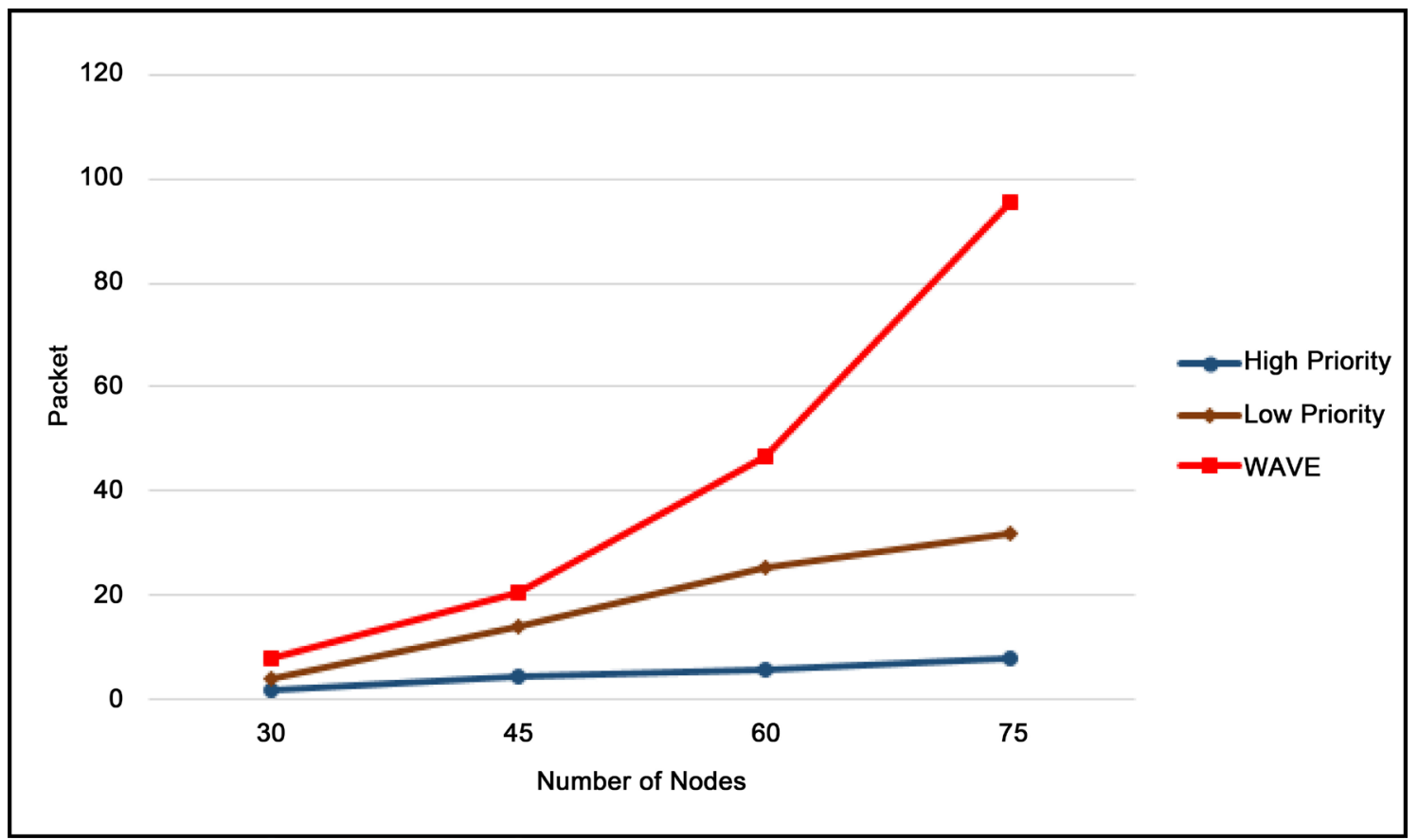

Figure 15. Inter-Cluster average queue length in high threshold. 


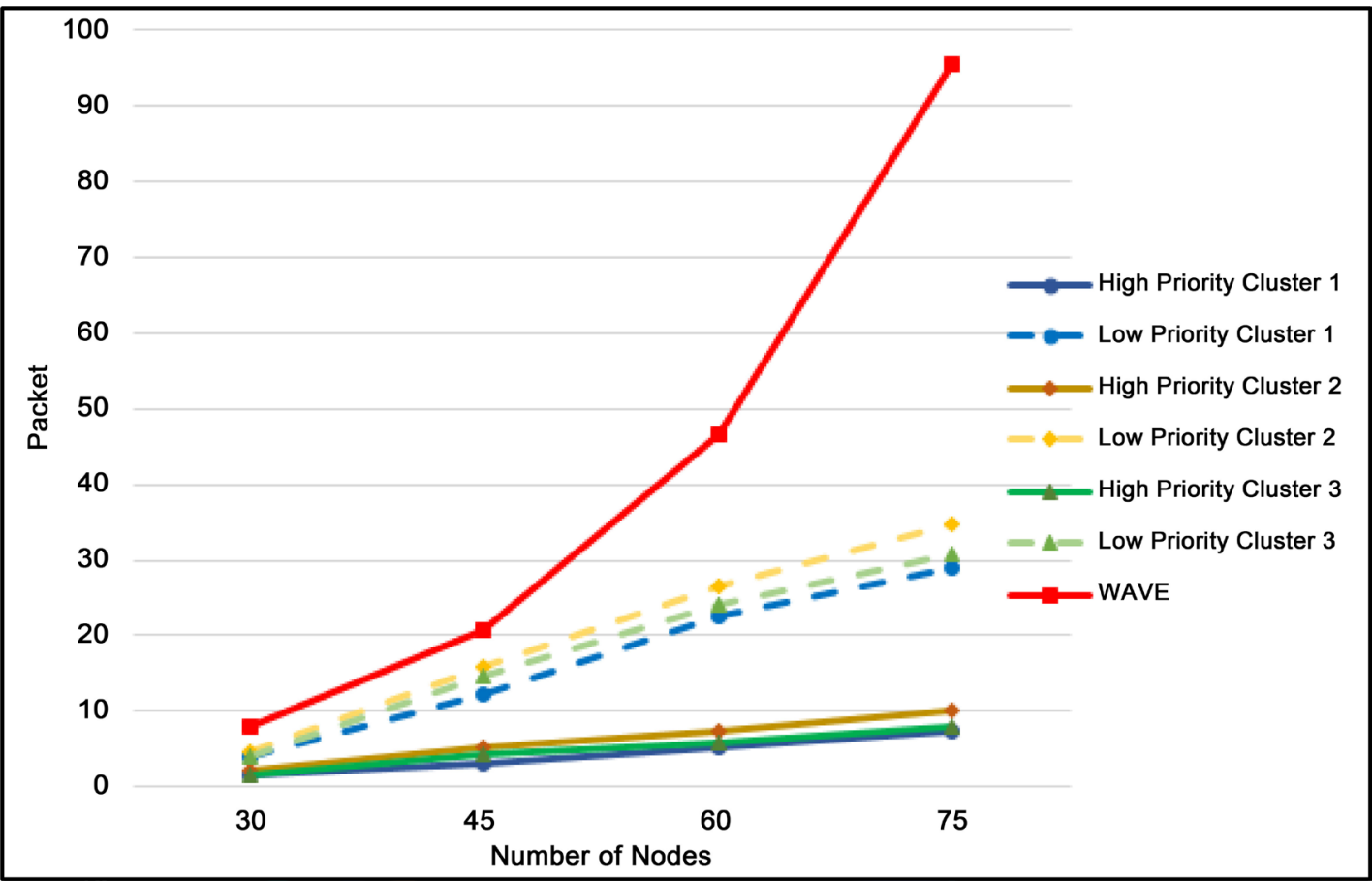

Figure 16. Average queue length of every cluster in high threshold.

lower queue length compare to WAVE.

Figure 16 illustrates the average length of the queue from every cluster in the high threshold of the simulation result. Cluster 2 has a higher average queue length compare with the other cluster in both of high priority and low priority simulations. The position of the cluster 2 is in between cluster 1 and cluster 3 on the topology. There are some moments where cluster 1 and cluster 3 need help from cluster 2's coordinator to communicate by forwarding the packet data. This causes the length of cluster 2's queue longer than the others do.

The result in Figure 17 illustrates that the average queuing delay increases as the number of nodes increase. WAVE suffers the longest delay from back off time that makes it cannot surpass the TDMA delay. WAVE even cannot outperform the low priority TDMA that been sacrificed for better performance of high priority TDMA.

Figure 18 illustrates the average delay of the queue from every cluster in the high threshold of the simulation result. Cluster 2 has a higher average delay compare with the other cluster in both of high priority and low priority simulations. The packet forwarding process experienced by the coordinator of the cluster 2 causes this. Member of cluster 2 needs to wait longer to get a turn to send packets.

As shown in Figure 19, packet loss increases as the number of nodes increase. WAVE performance falls on highest packet loss in a high number of nodes. In the low number of nodes, WAVE surpasses the total packet loss of TDMA but the packet loss increases rapidly along with increasing the number of nodes because of congestion. High priority TDMA showed the best performance by lowest packet loss, but the low priority TDMA experienced higher packet loss and 


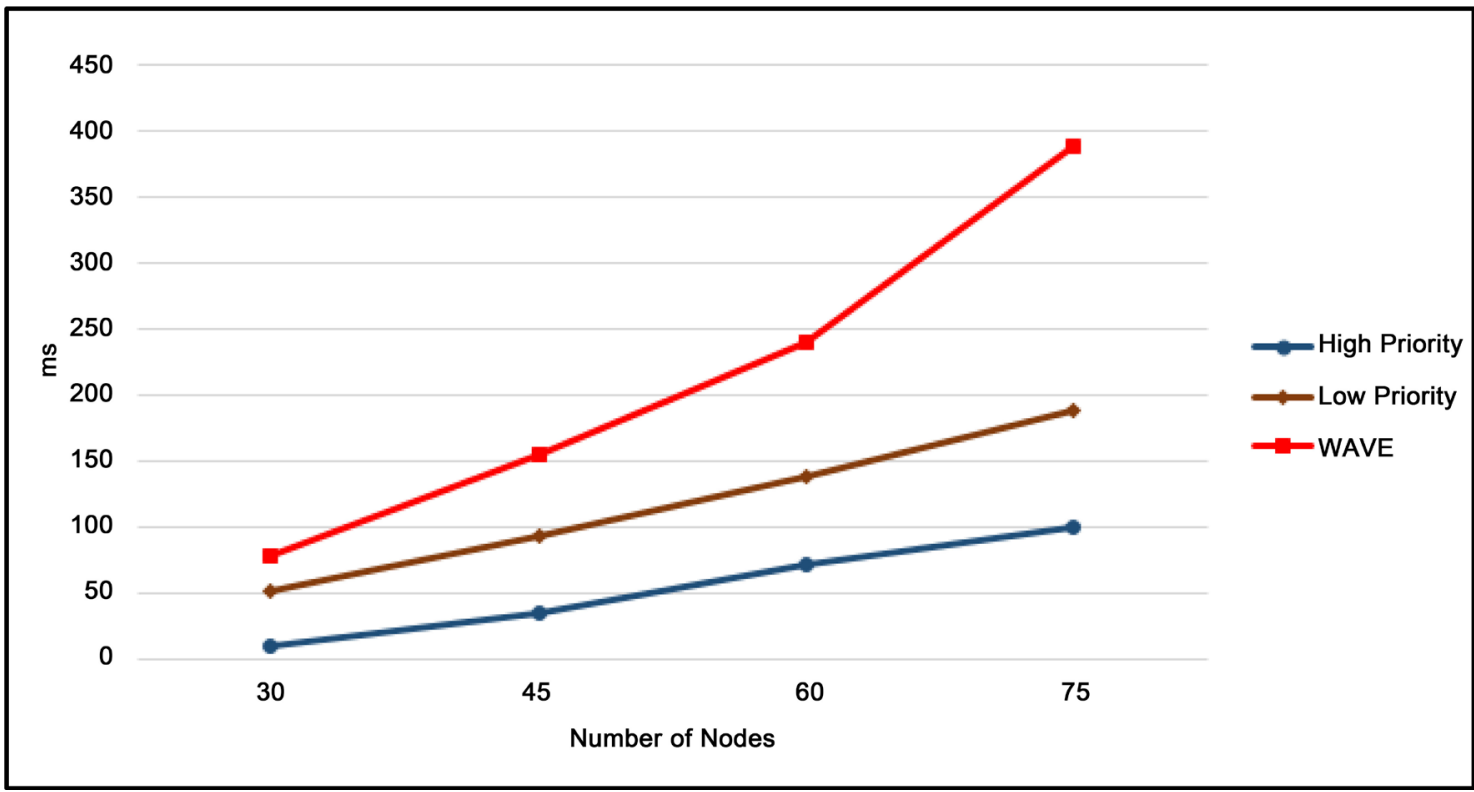

Figure 17. Average queuing delay in inter-cluster communications.

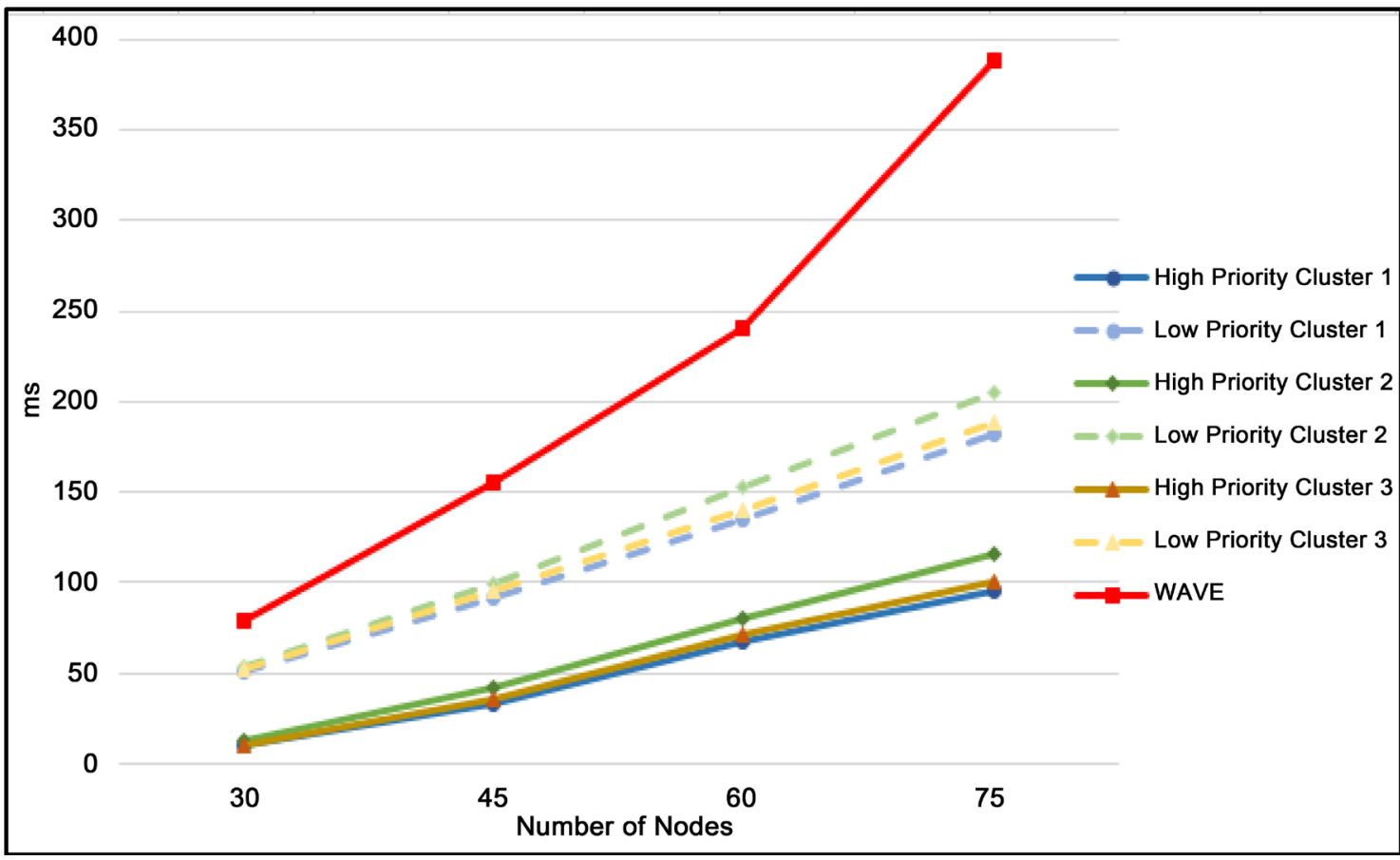

Figure 18. Queuing delay of every cluster in high threshold.

has better result comparing to WAVE when the number of nodes reaches 75 . This is happened because the low priority TDMA been sacrificed for better performance of the high priority TDMA.

The result in Figure 20 illustrates that the throughput decreases as the number of nodes increase. The performance of TDMA with high priority surpasses the number of nodes. The TDMA throughput decreases slowly along with the increasing of the number of nodes and approaching stationary condition. In the other hand, the WAVE throughput decreases from node 30 to 75 . 


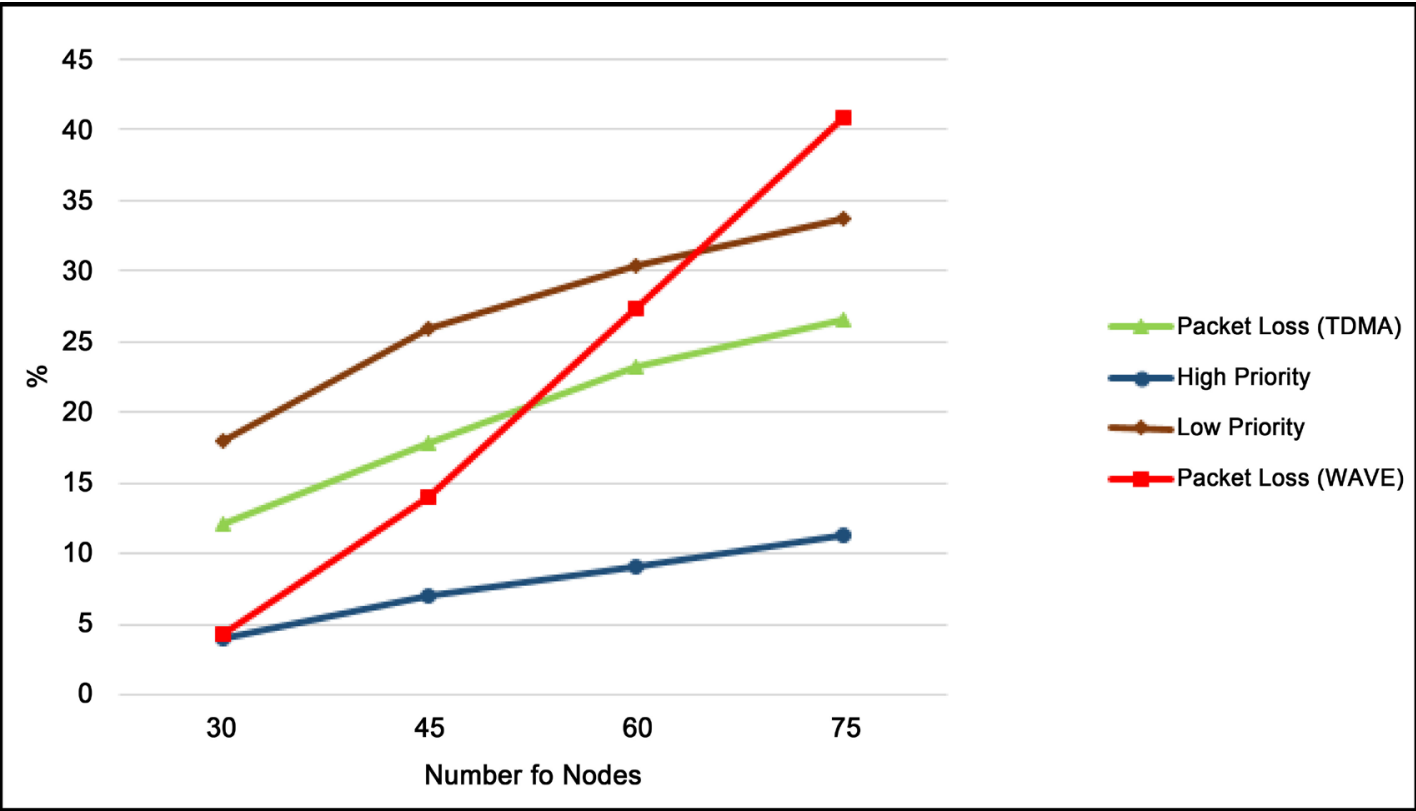

Figure 19. Packet loss ratio in inter-cluster communications.

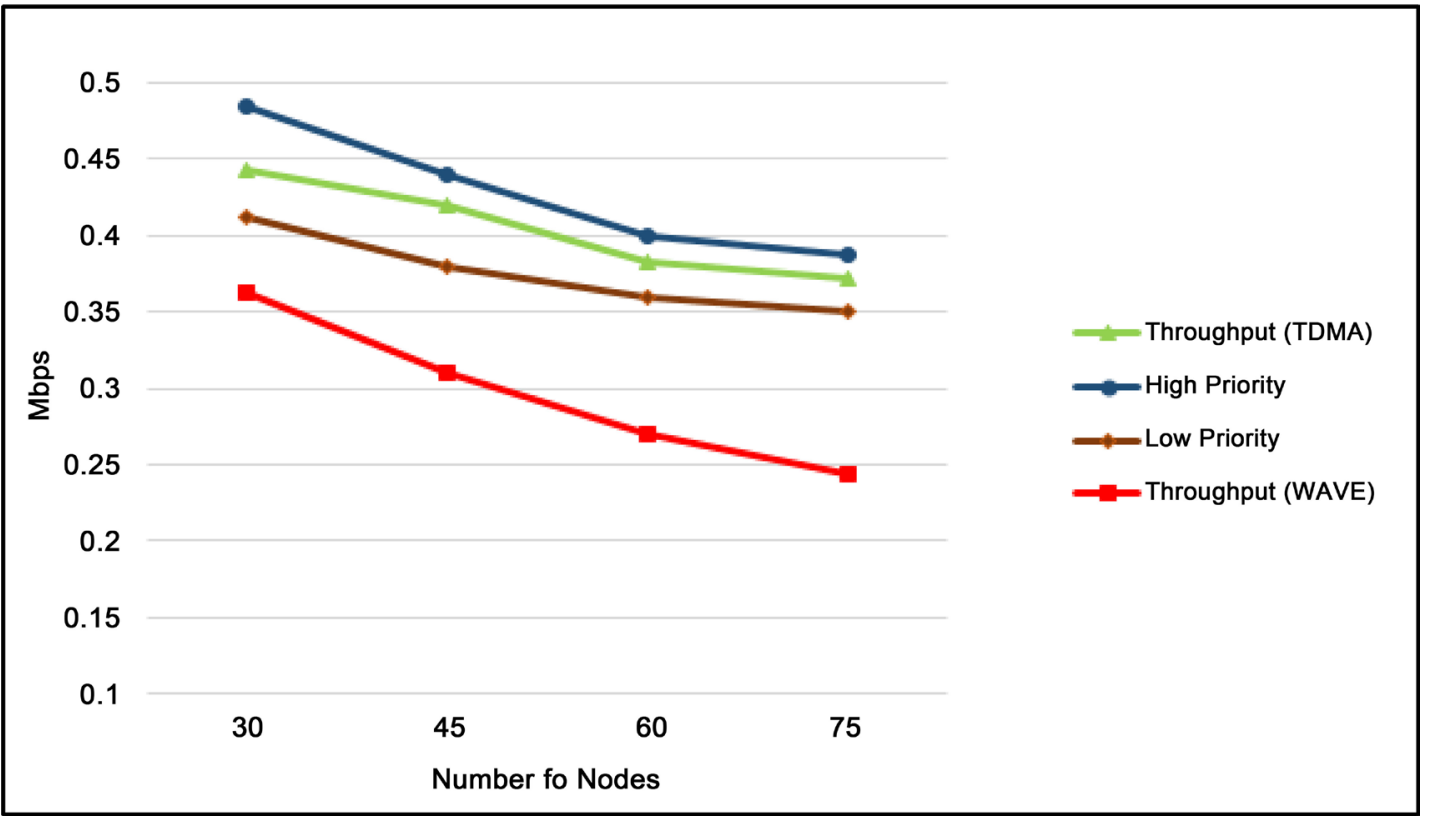

Figure 20. Throughput in inter-cluster communications.

Inter-cluster communication has lower average queue length and delay comparing to inter-cluster simulation result. This happened because of intra-cluster communication occurs more intense. If a node wants to do inter-cluster communication, the node needs to reserve a time slot of intra-cluster to send the packets to the coordinator before the coordinator forward it to the destination.

\section{Conclusions}

This journal proposed a new scheme to increase the TDMA performance using scheduling that assigned priority to the different types of traffic that separated it 
into two categories: high and low priority traffic. TDMA System will provide time slots based on traffic priority on a cluster-based communications. A new scheme to perform inter-cluster communications also proposed with reserving some part of the TDMA time slots for doing the forwarding between vehicle coordinator (VC). By giving priorities could significantly improve the performance of the traffic that considered as essential/important to be processed.

In intra-cluster communication, the proposed scheme has better performance compared to WAVE when the number of nodes is high. In inter-cluster communication, the proposed scheme has better performance compared to WAVE in queue length, delay, and throughput. In packet loss, when the number of nodes is low, WAVE surpasses the total packet loss of TDMA but the packet loss increases rapidly along with increasing the number of nodes.

\section{References}

[1] ASTM (2010) Standard Specification for Telecommunications and Information Exchange between Roadside and Vehicle Systems-5 GHz Band Dedicated Short Range Communications (DSRC) Medium Access Control (MAC) and Physical Layer (PHY) Specifications. E2213-03.

[2] Salvo, P., Turcanu, I., Cuomo, F., Baiocchi, A. and Rubin, I. (2016) LTE Floating Car Data Application Off-loading via VANET Driven Clustering Formation. 12th Annual Conference on Wireless On-Demand Network Systems and Services (WONS), Cortina d'Ampezzo, 20-22 January 2016, 1-8.

[3] Ucar, S., Ergen, S.C. and Ozkasap, O. (2015) Multihop-Cluster-Based IEEE 802.11p and LTE Hybrid Architecture for VANET Safety Message Dissemination. IEEE Transactions on Vehicular Technology, 65, 2621-2636.

[4] Uzcategui, R. and Acosta-Marum, G. (2009) Wave: A Tutorial. IEEE Communications Magazine, 47, 126-133. https://doi.org/10.1109/MCOM.2009.4939288

[5] Blot, G., Boucal, H., Rosseaux, F. and Saurel, P. (2016) An Experimentation of VANETs for Traffic Management. IEEE International Conference on Communications (ICC), Kuala Lumpur, 22-27 May 2016, 1-6.

[6] Midya, S., Majumder, K., Roy, A. and De, D. (2016) A Novel Vertical Handoff Scheme for Vehicular Ad-Hoc Networks. 3rd International Conference on Recent Advances in Information Technology (RAIT), Dhanbad, 3-5 March 2016, 117-122.

[7] Chang, C.-Y., Yen, H.-C. and Deng, D.-J. (2015) V2V QoS Guaranteed Channel Access in IEEE 802.11p VANETs. IEEE Transactions on Dependable and Secure Computing, 13, 5-17.

[8] Guo, G. and Wen, S. (2015) Communication Scheduling and Control of a Platoon of Vehicles in VANETs. IEEE Transactions on Intelligent Transportation Systems, 17, 1551-1563.

[9] Xu, K., Tripper, D., Qian, Y. and Krishnamurthy, P. (2015) Time-Dependent Performance Analysis of IEEE $802.11 \mathrm{p}$ Vehicular Networks. IEEE Transactions on Vehicular Technology, 65, 5637-5651.

[10] Dang, D.N.M., Dang, H.N., Nguyen, V., Htike, Z. and Hong, C.S. (2014) HERMAC: A Hybrid Efficient and Reliable MAC for Vehicular Ad Hoc Networks. IEEE 28th International Conference on Advanced Information Networking and Applications ( $A I N A)$, Victoria, 13-16 May 2014, 186-193.

[11] Nguyen, V., Oo, T.Z., Chuan, P. and Hong, C.S. (2015) An Efficient Time Slot Ac- 
quisition on the Hybrid TDMA/CSMA Multi-Channel MAC in VANETs. IEEE Communications Letters, 20, 970-973.

[12] Lin, J., Wu, C., Ohzahata, S. and Kato, T. (2014) A QoS Supporting Ad Hoc Network Protocol Combing Admission Based TDMA and 802.11 DCF. The 16th Asia-Pacific Network Operations and Management Symposium (APNOMS 2014), Hsinchu, 17-19 September 2014, 1-4.

[13] Rezazade, L., Aghdasi, H.S., Ghorashi, S.A. and Abbapour, M. (2011) A Novel STDMA MAC Protocol for Vehicular Ad-Hoc Networks. 2011 International Symposium on Computer Networks and Distributed Systems (CNDS), Tehran, 23-24 February 2011, 148-151.

[14] Nguyen, V., Dang, D.N.M., Jang, S. and Hong, C.S. (2014) E-VeMAC: An Enhanced Vehicular MAC Protocol to Mitigate the Exposed Terminal Problem. The 16th Asia-Pacific Network Operations and Management Symposium (APNOMS 2014), Hsinchu, 17-19 September 2014, 1-4. https://doi.org/10.1109/apnoms.2014.6996561

[15] Omar, H., Zhuang, W. and Li, L. (2013) VeMAC: A TDMA-Based MAC Protocol for Reliable Broadcast in VANETs. IEEE Transactions on Mobile Computing, 12, 1724-1736. https://doi.org/10.1109/TMC.2012.142

[16] Kawakami, T. and Kamakura, K. (2015) Modified TDMA-Based MAC Protocol for Vehicular Ad Hoc Networks. 2015 IEEE International Conference on Pervasive Computing and Communication Workshops (PerCom Workshops), St. Louis, 23-27 March 2015, 93-98.

[17] Bharati, S., Zhuang, W. (2013) CAH-MAC: Cooperative ADHOC MAC for Vehicular Networks. IEEE Journal on Selected Areas in Communications, 31, 470-479. https://doi.org/10.1109/JSAC.2013.SUP.0513042

[18] Zhang, T. and Zhu, Q. (2016) A TDMA Based Cooperative Communication MAC Protocol for Vehicular Ad Hoc Networks. 2016 IEEE 83rd Vehicular Technology Conference (VTC Spring), Nanjing, 15-18 May 2016, 1-6. https://doi.org/10.1109/vtcspring.2016.7504113

[19] Peng, F., Zhang, G., Huang, X., Ye, X. and Wu, M. (2015) A Novel TDMA-MAC Protocol for VANET Using Cooperative and Opportunistic Transmissions. IEEE 82 th Vehicular Technology Conference ( VTC Fall), Boston, 6-9 September 2015, 12. https://doi.org/10.1109/vtcfall.2015.7390806

[20] Jiang, X. and Du, D.H.C. (2016) PTMAC: A Prediction-Based TDMA MAC Protocol for Reducing Packet Collisions in VANET. IEEE Transactions on Vehicular Technology, 65, 9209-9223.

[21] Almalag, M.S., Olariu, S. and Weigle, M.C. (2012) TDMA Cluster-Based MAC for VANETs (TC-MAC). 2012 IEEE International Symposium on World of Wireless, Mobile and Multimedia Networks (WoWMoM), San Francisco, 25-28 June 2012, 1 6. https://doi.org/10.1109/wowmom.2012.6263796

[22] Almalag, M.S., El-Tawab, S., Olariu, S. and Weigle, M.C. (2013) A Modified TC-MAC Protocol for Multi-Hop Cluster Communications in VANETs. 2013 International Conference on Connected Vehicles and Expo (ICCVE), Las Vegas, 2-6 December 2013, 832-837. https://doi.org/10.1109/ICCVE.2013.6799909

[23] Shahin, N. and Kim, Y.-T. (2016) An Enhanced TDMA Cluster-Based MAC (ETCM) for Multichannel Vehicular Networks. International Conference on Selected Topics in Mobile \& Wireless Networking (MoWNeT), Cairo, 11-13 April 2016, 1-8.

[24] Sood, M. and Kanwar, S. (2014) Clustering in MANET and VANET: A Survey. 2014 International Conference on Circuits, Systems, Communication and Information Technology Applications (CSCITA), Mumbai, 4-5 April 2014, 375-380. 
https://doi.org/10.1109/cscita.2014.6839290

[25] Wang, H., Liu, R.P., Ni, W., Chen, W. and Collings, I.B. (2015) VANET Modeling and Clustering Design under Practical Traffic, Channel and Mobility Conditions. IEEE Transactions on Communications, 63, 870-881.

[26] Farooq, W., Khan, M. A. and Rehman S. (2016) A Cluster Based Multicast Routing Protocol for Autonomous Unmanned Military Vehicles (AUMVs) Communication in VANET. 2016 International Conference on Computing, Electronic and Electrical Engineering (ICE Cube), Quetta, 11-12 April 2016, 42-48.

[27] Wang, C., Li X., Li, F. and Lu, H. (2014) A Mobility Clustering-Based Roadside Units Deployment for VANET. 16th Asia-Pacific Network Operations and Management Symposium (APNOMS), Hsinchu, 17-19 September 2014, 1-6. https://doi.org/10.1109/apnoms.2014.6996595

[28] Zhang, R., Cheng, X., Yang, L., Shen, X. and Jiao, B. (2014) A Novel Centralized TDMA-Based Scheduling Protocol for Vehicular Networks. IEEE Transactions on Intelligent Transportation Systems, 16, 411-416.

[29] Sheu, T.-L. and Lin, Y.-H. (2014) A Cluster-Based TDMA System for Intervehicle Communications. Journal of Information Science and Engineering, 30, 213-231.

[30] Nguyen, V., Kim, O.T.T., Dang, D.N.M., Kim, S.S. and Hong, C.S. (2015) Application of the Lowest-ID Algorithm in Cluster-Based TDMA System for VANETs. 2015 International Conference on Information Networking (ICOIN), Cambodia, 12-14 January 2015, 25-30.

[31] Nguyen, V., Kazmi, S.M.A. and Hong, C.S. (2015) ECCT: An Efficient-Cooperative ADHOC MAC for Cluster-Based TDMA System in VANETs. International Journal of Distributed Sensor Networks, 2015, Article ID: 746438.

\section{Submit or recommend next manuscript to SCIRP and we will provide best} service for you:

Accepting pre-submission inquiries through Email, Facebook, LinkedIn, Twitter, etc. A wide selection of journals (inclusive of 9 subjects, more than 200 journals)

Providing 24-hour high-quality service

User-friendly online submission system

Fair and swift peer-review system

Efficient typesetting and proofreading procedure

Display of the result of downloads and visits, as well as the number of cited articles

Maximum dissemination of your research work

Submit your manuscript at: http://papersubmission.scirp.org/

Or contact jcc@scirp.org 